\title{
JOCHEN HÖRISCH
}

\section{„Seekrankheit auf festem Lande“ - Zur Krise der Literatursprache}

\begin{abstract}
Die Sprache der Literatur ist krisenfrei. Denn Dichtung ist bekanntlich - und bekanntlich heiBt: das ist seit Hesiod und Platon uralteuropäisches Basiswissen - nicht auf Wahrheit verpflichtet. Ihre Betriebslizenz, wenn sie denn erteilt wird, ist vielmehr das Recht zur systematischen Lüge. Was auch zur Folge hat, daß es wenig sinnvoll ist, literarische Aussage mit Sätzen zu bestreiten wie „Hans Castorp war doch gar nicht in Davos" oder "Madame Bovary hat ihren Mann doch gar nicht betrogen“. Schöne Literatur ist negations- und also ganz ungewöhnlich krisenimmun. Diese Einsicht in die Krisenimmunität der literarischen Sprache ist so alt und schlicht, daß sie ein wenig Brisanz erst gewinnt, wenn die elementare Verläßlichkeitskrise des Mediums Sprache so konstrastiv zum Modethema avanciert - also zu Beginn des 20. Jahrhunderts. Dichtung muß nämlich in gesteigerter Irritation erfahren, daß sie an dieser Krise nicht teilhat - gewissermaßen seit knapp dreitausendjähriger Wartezeit immer noch nicht an der so offen ausbrechenden Sprachkrise teilhat. Daß Dichtung lügt, ist seit ihren Anfängen unumstritten. Streiten kann man dann darüber, ob und ggf. unter welchen Rahmenbedingungen die systematische Lüge der Dichtung statthaft ist oder ob man die Dichtung, weil sie lügt, verbieten soll. Aber es wäre auch und gerade in der Zeit modemer Ausdifferenzierung von Funktionssystemen absurd ausgerechnet vom fiktionalen Medium Literatur zu erwarten, daß es richtige Bezeichnungen oder gar wahre Namen für Dinge. Sachverhalte und Zusammenhänge bereithält. Die Sprache der Literatur folgt, um modern und also systemtheoretisch zu reden, nicht dem Code richtig/falsch, sie ist vielmehr dem Code stimmig/nichtstimmig verpflichtet.
\end{abstract}

Ein junger Mann - so will es eine frühe Erzählung Franz Kafkas - geht tagtäglich in die Kirche. Nicht ein frommes Bedürfnis, sondern die Liebe zu einem Mädchen, das dort häufig Ruhe sucht, treibt ihn in den sakralen Bau. Sein Wunsch nach Nähe zu diesem Mädchen und nicht etwa nach der Nähe Gottes wird enttäuscht. Denn die junge Schöne bleibt aus. Statt des Mädchens trifft der junge Mann in der Kirche nun aber häufig auf einen ekstatischen Beter. Und dieser fasziniert ihn fast so sehr wie das vermißte Mädchen. Denn der Beter, der sich bei seinen „frommen Ausbrüchen" offenbar gerne von zahlreichen „zuschauenden Leuten“ umgeben 
läßt, scheint ein Geheimnis zu bergen, das der Beobachter ergründen will. Und so stellt er ihn eines Tages ohne Scheu vor Handgreiflichkeiten beim Verlassen der Kirche zur Rede. In deutlicher Anspielung auf die alttestamentarischen Worte Jacobs, der mit dem (Engel des) Herm um den göttlichen Segen rang', schreit er in einem pointiert nicht-biblischen Kontext. nämlich ,in den Lärm der vorüberfahrenden Straßenbahn: "Ich lasse Sie nicht. (...) Sie sind ein Glücksfang. Ich beglückwünsche mich.'”-

Der Beobachter ringt mit dem Beter - nicht etwa wie Jacob um Gottes Segen (den er auch kaum braucht, beglückwünscht er sich doch selbst). sondern aus einem deutlich geringer zu wertenden Motiv: weil er wissen will, warum der Beter eine so offenbare Lust daran habe, sich beim Beten beobachten zu lassen. „Wie benehmt Ihr Euch doch in der Kirche! Wie kann man andächtig sein, wenn man Euch anschauen muß." Die Reaktion auf diese Vorwürfe ist entwaffnend offen: „Ärgert Euch nicht, wenn ich sage, daß es der Z $Z$ weck meines Lebens ist, von den Leuten angeschaut zu werden." Grund zu einer gewissen Verärgerung gibt diese Antwort schon. Immerhin ist Kafkas kurze Erzählung 1904 entstanden (sie wurde erst fünf Jahre später veröffentlicht) - also zu Zeiten, in denen man noch nicht daran gewohnt bzw. medientechnologisch konditioniert war, z.B. den Papst televisionär beim Beten zu beobachten. Der namenlose Beter ist schon durch diesen Begriff 'Beter' als ein Sprechender charakterisiert. Aber eben als still Sprechender. Man kann ihm bei dem eigentümlich stillen Sprechakt, der da Beten heißt. zusehen. aber nicht verstehen geschweige denn sehen, was er zu Gott spricht.

Der Beobachter, der sein ursprüngliches Interesse am Mädchen offenbar völlig vergessen hat, reagiert auf dieses Geständnis des stillen Beters. $\mathrm{da} B$ er sich gerne als Objekt eines eigentümlichen Voyeurismus anbiete. geradezu überwertig gereizt. „'Was sagt $\mathrm{Ihr} \mathrm{da}$ ', rief ich viel zu laut .... aber ich fürchtete mich dann, die Stimme zu schwächen. 'wirklich was sagtet Ihr da. Ja ich ahne schon, ja ich ahnte es schon, seit ich Euch zum erstenmal sah. in welchem Zustande Ihr seid. Ich habe Erfahrung und es ist nicht scherzend gemeint, wenn ich sage, daß es eine Seekrankheit auf festem Lande ist. Deren Wesen ist so, daß Ihr den wahrhaftigen Namen der Dinge vergessen habt und über sie jetzt in einer Eile zufällige Namen

1. Mose 32, 24-26 (Luther 1546): „Da rang ein Man mit jm bis die morgenröte anbrach. Vnd da er sahe / das er jn nicht vbermocht / rüret er das Gelenck seiner hüfft an / Vnd das gelenck seiner hüfft ward vber dem ringen mit jm / verrenckt. Vnd er sprach / Las mich gehen / denn die morgenröte bricht an / Aber er antwortet / Jch las dich nicht / du segenest mich denn."

: F. Kafka: Gespräch mit dem Beter. In: Erzählungen, ed. M. Müller. Stuttgart 1995. S. 11. Zu Kafkas Sprachreflexionen cf. K. Pape: Sprachkunst und Kunstsprache bei Flaubert und Kafka. St. Ingbert 1996. 
schüttet. Nur schnell, nur schnell! Aber kaum seid Ihr von ihnen weggelaufen, habt Ihr wieder ihre Namen vergessen. Die Pappel in den Feldern, die Ihr den 'Turm von Babel' genannt habt, denn Ihr wußtet nicht oder wolltet nicht wissen, daß es eine Pappel war, schaukelt wieder namenlos und Ihr müßt sie nennen 'Noah, wie er betrunken war'.' / Ich war ein wenig bestürzt, als er sagte: 'Ich bin froh, daß ich das, was Ihr sagtet, nicht verstanden habe.' / Aufgeregt sagte ich rasch: 'Dadurch, daß Ihr froh seid darüber, zeigt $\mathrm{Ihr}$, daß Ihr es verstanden habt.".

Die textimmanente Warnung ist unüberhörbar. Wer versucht, diesen rätselhaften Wortwechsel zu verstehen und in hermeneutisches Kleingeld zu transferieren, geht das Risiko ein, sich lächerlich zu machen. Die funktionale Problemlage aber, die von dieser Textpassage fokussiert wird, läßt sich immerhin angeben und analysieren. Ersichtlich geht es in dem zitierten Text, der als ein herausragendes Beispiel für die um 1900 besonders virulente Krise eben nicht so sehr der Literatursprache, sondern der Spracherfahrung überhaupt gilt, um die Unverläßlichkeit des Mediums Sprache. Aber diese verbreitete Feststellung ist, um die berühmte Wendung aus Poes Erzählung >The purloined letter` zu zitieren, ,a little bit too obvious." $\mathrm{Da}$ wir seit dem Kollaps des Turms zu Babel die wahren Namen der Dinge vergessen haben, daß Pfingstereignisse (um zurückhaltend zu formulieren) selten und also nicht institutionalisierbar sind daß die Sprache nichts als ein vagabundierendes Heer von Metaphern und Metonymien ist, daß wir in der sprachlich gedeuteten Welt nicht sehr verläßlich zu Haus sind, daß Worte wie modrige Pilze im Munde zerfallen können, daß Signifikanten arbiträr sind - all das ist spätestens seit Nietzsche, Mauthner, de Saussure, Rilke und Hofmannsthal theoretisch-literarische Basiseinsicht. Kafka wäre nur einer von vielen Sprachskeptikern um 1900, wenn sein Text nicht mehr Aufschluß gäbe. Der erfahrene Beobachter in seiner Erzählung ist mit der Diagnose des Problems, das ein anderer hat, ja auch bemerkenswert schnell (ein wenig zu schnell) zur Hand. „Ich habe Erfahrung - es ist eine Seekrankheit auf festem Lande - Ihr habt die wahrhaftigen Namen der Dinge vergessen." Das aber: daß wir für die Dinge und Sachverhalte keine wahrhaftigen Namen, sondern nur arbiträre und konventionelle Zeichen haben, ist eine noch weniger originelle Einsicht. Denn sie datiert nicht aus der Zeit um 1900, sondern ist einige Jahrtausende älter, wie die witzige Anspielung auf den Turmbau zu Babel umgehend anzeigt. Was folgt, ist eine durch bloße Assonanzen und Bildähnlichkeiten (wie Turm - hoher Baum - Schwanken im Wind - Schwanken bei Trunkenheit) zusammengehaltene Assoziationskette, an der Freud seine helle Freude hätte. Diese Ton-Bild-Kette ist sachlich hochheikel, je-

Kafka: I.c., p. 12. 
doch in sich stimmig. Denn sie führt über die Wörter „Pappel“ und „Babel" zum Gebabbel des trunkenen Noah, der sein Geschlecht nicht den Blicken seiner Söhne entzieht $t^{4}$ - sich also so problematisch den Blicken darbietet wie der Beter.

Dargeboten aber wird somit auch ein Problem, das mehr Aufmerksamkeit verdient als das spätestens seit Nietzsches epochalem Essay ,Über Wahrheit und Lüge im außermoralischen Sinner (er entstand schon 1873. wurde aber erst 1903 posthum publiziert) überdeutliche Problem der Unverläßlichkeit von Sprache. Nämlich - ich komme zu meiner ersten These - das Problem, daß die Literatursprache genau dieses Problem nicht hat. Die Sprache der Literatur ist krisenfrei. Denn Dichtung ist bekanntlich - und bekanntlich heißt: das ist seit Hesiod und Platon uralteuropäisches Basiswissen ${ }^{5}$ - nicht auf Wahrheit verpflichtet. Ihre Betriebslizenz. wenn sie denn erteilt wird ist vielmehr das Recht zur systematischen Lüge. Was auch zur Folge hat, daß es wenig sinnvoll ist, literarische Aussage mit Sätzen zu bestreiten wie .Hans Castorp war doch gar nicht in Davos" oder "Madame Bovary hat ihren Mann doch gar nicht betrogen". Schöne Literatur ist negations- und also ganz ungewöhnlich krisenimmun. Diese Einsicht in die Krisenimmunität der literarischen Sprache ist so alt und schlicht, daß sie ein wenig Brisanz erst gewinnt, wenn die elementare Verläßlichkeitskrise des Mediums Sprache so kontrastiv zum Modethema avanciert - also zu Beginn des 20. Jahrhunderts. Dichtung muß nämlich in gesteigerter Irritation erfahren, daß sie an dieser Krise nicht teilhat - gewissermaßen: seit knapp dreitausendjähriger Wartezeit immer noch nicht an der so offen ausbrechenden Sprachkrise teilhat. Daß Dichtung lügt, ist seit ihren Anfängen unumstritten. Streiten kann man dann darüber, ob und ggf. unter welchen Rahmenbedingungen die systematische Lüge der Dichtung statthaft ist oder ob man die Dichtung. weil sie lügt. verbieten soll. Aber es wäre auch und gerade in der Zeit moderner Ausdifferenzierung von Funktionssystemen absurd, ausgerechnet vom fiktionalen Medium Literatur zu erwarten, daß es richtige Bezeichnungen oder gar wahre Namen für die Dinge, Sachverhalte und Zusammenhänge bereithält. Die Sprache der Literatur folgt. um modern und also systemtheoretisch zu reden, nicht dem Code richtig/falsch; sie ist vielmehr dem

4 1. Mose 9, 20-23 (Luther 1546): „Noah aber fieng an vnd ward ein Ackerman vnd pflantzte Weinberge. Vnd da er des Weins tranck; ward er trunkken ; vnd lag in der Hütten aufgedeckt. Da nun Ham / Canaans vater / sahe seines Vaters scham / saget ers seinen beiden Brüdern draussen. Da nam Sem vnd Japhet ein Kleid / vnd legten es auff jre beide Schulder / vnd gingen rücklings hin zu / vnd deckten jres Vaters scham zu."

" Cf. Heinz Schlaffer: Poesie und Wissen - Die Entstehung des ästhetischen Bewußtseins und der philologischen Erkenntnis. Frankfurt/M. 1990 
Code stimmig/nicht-stimmig verpflichtet. Und die Assoziationskette Babel-Gebabbel-Pappel-Schwanken-Noah-Unsagbares-bzw.-Unerhörtessehen ist sachlich unhaltbar und poetisch hochgradig stimmig.

Krisen eines Mediums oder eines Funktionssystems kann es nur geben, wenn selbst- oder fremdgesetzte Ansprüche nicht erfültt werden. Literatur aber erfüllt ihren eigenen und den an sie herangetragenen Anspruch glänzend den Code wahr/falsch zugunsten des Codes stimmig/unstimmig zu unterlaufen. Und also ist die Sprache der Literatur krisenfrei - so krisenfrei, wie nach der berüchtigten These des platonischen Kratylos alle Rede, die nicht mehr zu leisten behauptet als eben dies: zu sagen, was sie sagt, Aussage und eben nicht Transportmittel für Ausgesagtes zu sein. Auf den Turmbau zu Babel verweist Kafkas Erzählung ausdrücklich. Ihr aufund anregenderer Subtext aber ist die These des Kratylos von der krisenfreien Kraft einer Sprache, in der ,alle Worte und Benennungen gleich richtig sind" - einer Sprache. die Sagen und nicht Etwas-Aussagen, die Sprechakt und nicht Vermittlung eines propositionalen Gehalts ist. Sokrates ist irritiert - so irritiert wie in keinem zweiten platonischen Dialog. Er, der von sich sagt ,Ja, guter Kratylos, ich wundere mich selbst schon lange über meine eigene Weisheit und kann kaum daran glauben" unterwirft die kratylistische These, daß es keine falschen Benennungen gebe, einer strengen Prüfung, indem er eine ganze Reihe von Wortklassen kasuistisch analysiert und immer zum selben erzvernünftigen, eben sokratischen Ergebnis kommt: die These des Kratylos von der grundsätzlichen Richtigkeit der Sprache ist unhaltbar. Selbst für den Sonderfall der Namen, die doch in einem besonders innigen Verhältnis zum Benannten zu stehen scheinen, trifft sie nicht zu. .Sokrates: Lügt auch etwa nicht einmal derjenige, welcher sagt, er heiße Hermogenes? Daß nur nicht am Ende auch das nicht möglich ist: zu sagen, er sei Hermogenes, wenn er es nicht ist? / Kratylos: Wie meinst du das? / Sokrates: Ob dies etwa, daß man überhaupt nichts Falsches sagen könnte. ob dies der Gehalt deines Satzes ist? Denn gar manche behaupten dies, lieber Kratylos, jetzt und auch sonst schon. / Kratylos: Wie sollte denn auch, Sokrates, wenn einer doch das sagt, was er sagt, er nicht etwas sagen, was ist? Oder heißt das nicht eben Falsches reden (pseudä legein): sagen, was nicht ist (to me ta onta legein)?" / Sokrates: Dieser Satz, Freund ist für mich und für mein Alter zu hoch."'

So viel fröhliche Krisenjen- oder wohl eher: -diesseitigkeit ist nicht nur Sokrates suspekt. Dabei läßt sie sich problemlos in linguistisches Neudeutsch transformieren: wenn einer sagt, was er sagt, sagt er etwas, was

- Platon: Kratylos. In: Werke in acht Bdn griech-dt., ed. G. Eigler, Bd. 3. Darmstadt 1990, S. 539 (429c,d). 
ist, weil es statthat - nämlich einen Sprechakt. Sprechakte sind Sprechakte sind Sprechakte und können als Sprechakte nicht falsch sein. Man kann ein Versprechen brechen; die durch den Sprechakt eines Standesbeamten gestiftete Ehe kann scheitern: der Urteilsspruch eines Gerichtes kann durch eine Revision angefochten werden. Und doch gilt, daß diese Stiftungs-Sprechakte als Sprechakte, die etwas stiften, sagen. daß sie etwas stiften und also sagen, was ist. Diese weitreichende und tiefgründige Banalität gilt selbstredend für alles Gesagte und Geschriebene: als Sprechund Schreibakte sagen und schreiben sie, was sie sind. 'O gegrapha gegrapha: quod scripsi, scripsi; quod dixi. dixi!'. Damit sagen Sprech- und Schreibereignisse zugleich, daß sie als solche nicht falsch sein können. Falsch können nur Propositionen sein, sofern man sie im Hinblick auf ihren propositionalen Gehalt überprüft. Die Sprache der Literatur aber besteht nicht aus Propositionen'. die irgendeinem wie immer auch zu definierenden „realistischen“ Adäquatheitskritierium, sondern nur einem Stimmigkeitskriterium unterliegen. Und so ist die Literatursprache der Möglichkeit beraubt, in fundamentale Krisen zu stürzen. Wenn bei IDSVorträgen zu kalauern erlaubt ist: die Sprache der Literatur stiftet ihre Aussagen und geht dann stiften. Um mit einem banalen und doch für viel Aufregung sorgenden Beispiel zu illustrieren: wenn Stefan Hermlin in einem Verwaltungsfragebogen angibt, im $\mathrm{KZ}$ gewesen zu sein, ist das eine falsche Angabe. Wenn er in seiner gattungsmäßig schwer einzuordnenden. jedenfalls belletristischen Prosa .,Abendlicht" dasselbe schreibt und angibt, ist das Literatur. Wie abgründig oberflächlich es um das Verhältnis von Wahrheit und Sprache bestellt ist, weiß schon jene weise Kinderfrage: „Kanst du die Wahrheit sagen? - Ja! - So sag sie doch! - Irritiertes Schweigen. - Ätsch: 'Die Wahrheit!'” Man kann dieselbe Einsicht auch in anderem genus dicendi vortragen. Etwa so wie in den Versen Stefan Georges. die schon der letztjährige Duden-Preisträger Helmut Henne. umsichtig die Nietzsche- und die Duden-Linie der Sprachbetrachtung auslotend, für seine ..sprachliche Erkundung der Moderne" herangezogen hat: .Worte trügen worte fliehen i Nur das lied ergreift die seele."

Kurzum: die Sprache der Literatur ist von elementarer Krisenunanfälligkeit. Und eben das macht ihre Dauer-Krise aus. Denn nichts ist so langweilig wie Krisenabstinenz. Vor allem für Literatur. die bekanntlich zweierlei soll: aut prodesse aut delectare. Belehren kann man nur, wenn

Der Verweis auf die klassischen phänomenologischen Untersuchungen von Roman Ingarden (Das literarische Kunstwerk. Tübingen 1972, bes. p. 191 und Fn p. 236) und Käte Hamburger (Die Logik der Dichtung. Ffm/Berlin/Wien 1980) ist hier obligatorisch.

* Zit. und kommentiert bei H. Henne: Sprachliche Erkundung der Modeme. (Rede des Preisträgers zum Duden-Preis 1996). Mannheim 1996. S. 27 
man Neues sagt. Neues aber ist per definitionem eine Krise des Alten. Und erfreuen oder gar erregen kann man nur, wenn man Krisentaugliches erzählt. Man kann es auch so banal wie Reich-Ranicki sagen: Literatur darf nicht langweilig sein. Und deshalb - so meine zweite These - verschreibt sich das auffallend krisenfreie Sprachmedium, das da Literatur heißt, selbst systematisch Krisen. Literatur prozediert als Symptomverschreibungsmedium. Unheilbar gesund mag und darf Literatur nicht sein. Und so sucht sie angestrengt nach Möglichkeiten, sich Blessuren zu holen. Beliebte Möglichkeiten dafür sind 2. B. Grenzüberschreitungen zwischen Diskursgattungen: Literatur tut so, als könne sie sachlich mitreden. Und deshalb legt sie sich gerne mit Spezialistendiskursen an und bestreitet z. B. Kernphysikern, daß sie Murphys Gesetz (was schiefgehen könne, gehe häufiger schief, als die Wahrscheinlichkeitsrechnung es nahelege) angemessen berücksichtigt haben oder IDS-MitarbeiterInnen, daß die neue Orthographiereform sinnvoll ist. Die für unsere Themenstellung spannendere Möglichkeit ist, besonders stark auf den poesiespezifischen Code stimmig/unstimmig zu setzen und z. B. für einen besonders reizvollen Reim jede sachliche Plausibilität der Aussage für irrelevant zu erklären. Dann kann man so schöne und - in soziologischer Perspektive unsinnige - Verse schmieden wie Gottfried Benn es tat: „Der soziologische Nenner, / Der hinter Jahrtausenden schlief, / Heißt: ein paar große Männer / Und die litten tief."

Die Sprache der Literatur kann sich Krisen auch verschreiben, indem sie Referenz auf andere Literatur oder auf Eigentümlichkeiten einer Sprache (wie z. B., daß es im Deutschen kein Reimwort auf den ja nicht ganz unwichtigen Begriff 'Mensch' gibt) für wichtiger hält als das, was man Weltreferenz nennen könnte. Von Peter Rühmkorf stammen die prächtigen Verse, die sich zur Illustration dieser poetischen Disposition besonders gut eignen: „Die schönsten Gedichte der Menschen - / Nun finden Sie mal einen Reim! - / Sind die Gottfried Bennschen: / Hirn, lernäischer Leim / Sinkende Euphratthrone, / Rosen auf Rinde und Stamm - / Gleite Epigone / Ins süße Benn-Engramm." Der Einfallsreichtum des Mediums Literatur bei ihrem steten Bemühen, auch ordentlich krisengeschüttelt zu sein. ist gro $\beta$ und kann hier naturgemä $\beta$ nicht erschöpfend behandelt werden. Denn es bleibt bei der These von der abgründigen Krisenunanfälligkeit der Literatursprache. Die Sprache der Literatur kann so esoterisch bis unverständlich sein, wie sie will; Literatur kann die Linearität der Schrift aufsprengen und in mehreren Kolumnen schreiben (wie Joyce, Arno Schmidt oder Derrida es vorexerziert haben); sie kann lallen oder beleidigen; sie kann (wie die Klingklanglyrik von Thomas Kling oder Jirgls die deutsch-deutsche Grenze ebenso wie die Grenzen von Oralität und Schriftlichkeit auslotenden Roman ,Abschied von den feinden() ihre eigenen Orthographieregeln oder unerhört neue Worte erfinden, die keiner 
hören oder lesen will; sie kann ihre interne Stimmigkeit sogar in extremer Dissonanz und Unstimmigkeit suchen - sie bleibt doch unrettbar gesund. Um auf Kafkas Worte zurückzukommen: die Krise der Literatursprache war, ist und bleibt eine "Seekrankheit auf festem Lande".

Festen Boden haben wir nie unter den Füßen; die Erde dreht sich bekanntlich mit einer Geschwindigkeit, die es erklärungsbedürftig macht. warum wir nicht ständig megaseekrank sind. Die Literatur hat von den Abgründigkeiten. Kreiselbewegungen und Unverläßlichkeiten des Mediums Sprache ein elementares Bewußtsein. Das macht sie so stabil und krisenunanfällig: in der Sprache der Literatur gerät die Krise der Sprache in eine Krise. Und diese Dialektik versetzt sie in die Lage zu beobachten, wie liebenswürdig unsinnig alle Versuche sind dem Medium Sprache etwa durch sprachanalytischen Exorzismus oder durch das, was Derrida den .Terrorismus des Konsenses" nannte - Stabilität zu verleihen. Die Frage nach stabilen Beziehungen zwischen Wirklichkeit und Sprache bzw. zwischen Sein und Sinn ist eine Kinderfrage - und von der peinlichen Unvermeidbarkeit aller Kinderfragen. Es ist, wie das Medium Literatur erkennt, ein Grundirrtum, ihre Lösung ausgerechnet Sprachphilosophen, Linguisten oder Philologen anzuvertrauen. Denn diese können immer nur dasselbe herausfinden - nämlich: daß kratylistische Programme aller Art außerhalb der Literatur zum Scheitern verurteilt sind. ${ }^{9}$ Für intersubjektiv einigermaßen verläßliche Korrelationen zwischen Sein und Sinn sorgt eben nicht das Medium Sprache, das ja auch nicht auf Identitäten und Korrelationen. sondern vielmehr auf Dissenz und Differenz spezialisiert ist.

Für die ontosemiologische (also Sein auf Sinn. Ontologie auf Semiologie et vice versa beziehende) Suggestion. Sein sei sinnvoll und Sinn sei vorhanden, sorgen vielmehr die ,shared symbolic systems" (Talcott Parsons). die Gesellschaften zusätzlich zum Krisenmedium Sprache als verbindliche bis funktional unvermeidliche Massenmedien emergieren lassen. um basale soziale Synthesis zu garantieren. Die Literatur der Neuzeit, der Moderne und der Postmoderne hat für unsere sog. abendländische Kulturtradition vor allem drei ontosemiologische Leitmedien fokussiert: das Abendmahl, das Geld und die neuen AV-Medien." Sie organisieren medientechnologisch den Rahmen, in dem Korrelationen von Sein und Sinn geschaltet und intersubjektiv akzeptiert werden. Die großen literarisch reflektierten Sprachkrisen um 1600 und 1900 sind deshalb weniger als interne Krisen des Mediums Sprache, sondern vielmehr als Epiphäno-

- Zur hartnäckig anhaltenden Nachwirkung der Kratylos-These cf. G. Genette: Mimologiken - Reise nach Kratylien. München 1996 (Paris 1976).

11) Hierzu J. Hörisch: Brot und Wein - Die Poesie des Abendmahls. Frankfurt M 1992 und ders.: Kopf oder Zahl - Die Poesie des Geldes. Frankfurt/M. 1996. 
mene ontosemiologischer Erosionen zu verstehen. Wenn Hamlet auf die Frage „Prince, what do you read?" die grandiose Antwort gibt: „words, words, words"; wenn Don Quixote lernen muß, daß zwischen dem Buch der Welt und der Welt der Bücher keine verläßlichen Beziehungen herrschen; oder wenn Emblemata die Zuordnungen von pictura und scriptio problematisieren - so hat das wenig mit der schon damals uralten und vertrauten Krise des Mediums Sprache, sehr viel aber mit der postreformatorischen Erosion des Glaubens zu tun, daß das Wort Fleisch ward und mitten unter uns lebte und daß die Transsubstantiation von Sein in Sinn et vice versa sich unendlich reproduzieren läßt und jeden Sonntag, den Gott werden läßt, neu gelingt. Europa stellt um 1600 vom Leitmedium Eucharistie auf das seltsam oblatenähnliche Leitmedium Geld um. Wenn 300 Jahre später (dadurch, daß er seine Worte Lord Chandos, also einem Zeitgenossen Shakespeares in den Mund legt, schlägt Hofmannsthal selbst die Brücke zwischen den Sprachkrisen um 1600 und um 1900) die Worte wie modrige Pilze im Munde zerfallen, so auch deshalb, weil das neue ontosemiologische Leitmedium Geld altert und von einer neuen Formation beerbt wird: der Formation der AV-Medien. Natürlich müssen auch poetischmoderne Worte modern (modern/modern), wenn Fotografien und Filme das Reale besser speichern und wiedergeben können als Schrift es je vermochte."

Zurück zur Kafka-Formel von der „Seekrankheit auf festem Lande“! Mit dieser Formel hat es ersichtlich (und nicht etwa unüberhörbar) eine eigentümliche Bewandmis. Ist das Wort „Seekrankheit“ doch völlig homophon mit dem Wort "Seh"- im Sinne von „Augenkrankheit". Die ergänzende Wendung ,auf festem Lande“ und der unmittelbare Kontext, der so deutlich vom Wortfeld "schwanken“ dominiert wird, lassen die auf Homophonie abhebende Lesung ,Sehkrankheit" erst einmal unplausibel erscheinen. Und doch: daß der stille Beter es zum „Zweck (s)eines Lebens“ erklärt, „von den Leuten angeschaut zu werden“, und daß Kafkas Erzählung so deutlich die visuelle mit der kommunikativen Sphäre kontrastiert, macht diese Lesung legitim. Überdies hat Kafka das Problem des Kontrastes zwischen Wahrnehmung und Kommunikation ja in sein Schlüsselwort hineinkopiert: die Differenz von See- und Sehkrankheit läßt sich eben nur sehen, nicht aber hören. Und damit bin ich bei meiner dritten These: weil die Sprache der Literatur krisenfrei ist, traut sie sich eine ungeheure Aufgabe zu. Nämlich: Wahrnehmungen kommunizierbar zu machen. Der Unsagbarkeitstopos begleitet Literatur seit ihren Anfängen. Zumeist wird er mit erhabenen Qualitäten versehen. Erhaben ist (nach F. Lyotards bündi-

"Zu Hofmannsthals Reaktion auf die neuen Medien cf. Uwe C. Steiner: Die Zeit der Schrift - Die Vergänglichkeit der Schrift und die Vergänglichkeit der Gleichnisse bei Hofmannsthal und Rilke. München 1996. 
ger Kant-Exegese), was sich der Repräsentation und Darstellung entzieht. Wenn Leidens- oder Glückserfahrungen so erhaben sind, daß nur poetische Sprache sie auszudrücken vermag. wenn ein Gott einem Dichter zu sagen gibt. was und wie er leidet oder genießt, dann scheint der Unsagbarkeitstopos erfüllt zu sein. Wer so argumentiert, übersieht geradezu systematisch das schlichte und eben nicht erhabene systematische Problem, das zu lösen die Literatursprache antritt: das zu sagen. was einfach deshalb unsagbar ist, weil es wahrgenommen und eben nicht kommuniziert wurde.

Lassen Sie mich dieses Problem an einem Text illustrieren, der auf der Schwelle der literarischen Moderne steht ${ }^{12}$..Er wußte. daß es da eine Geschichte gab von einem Mann ohne Schatten." So heißt es in H.C. Andersens 1847 entstandener Geschichte .Der Schatten“ (,.Skyggen") ".3 von einem Gelehrten, der eben nicht umsonst Gelehrter ist. Er trägt schwer an dem Wissen der Vorwelt. Und so weiß er, als auch er in einem heißen Land seinen Schatten verliert, wieviel Mühe er sich geben muß bzw. wieviel Mühen ihm noch bevorstehen, wenn er bei seiner Heimkehr noch eine originelle Geschichte erzählen will. Denn die Leute in seiner Heimat würden ansonsten sagen. „daß er herging und nachahmte, und das hatte er nicht nötig." Der Gelehrte will keine Geschichte haben, die ein bloßer Schatten von Peter Schlemihls Schattengeschichte wäre.

Der Preis für diese Originalitätssucht wird hoch sein. Zwar scheint sich vorerst die Lage wunderbar zu lichten. Dem Gelehrten wächst nämlich flugs ein neuer Schatten nach. Er kehrt in sein nördliches Heimatland zurück und schreibt wie zuvor ,Bücher über das Wahre in der Welt. über das Gute und über das Schöne." Doch damit nicht genug. Nach Jahren kehrt auch sein alter Schatten zurück. Dem ist es, wie seine elegante Kleidung bezeugt, in der Zwischenzeit offenbar gut gegangen. Nun will er sich vom ehemaligen Herrn freikaufen und seinen Weg zur Menschwerdung vollenden. Und so hätte der Gelehrte in deutlicher Umkehrung der SchlemihlGeschichte beides: einen neuen Schatten und viel Geld. Doch der Gelehrte will kein Geld. sondern, wie es sich in der Neuzeit für einen rechten Gelehrten gehört, ungewöhnliche Geschichten mit hohem Neuigkeitswert. Und die kann der Ex-Schatten versprechen: „Ein Mann ein Wort! / Ein Wort ein Schatten!"

Ungewöhnlich ist die Geschichte gewiß. Denn der Schatten kam seinem Herrn auf buchenswerte Weise abhanden. An den Abenden. an denen

1- Das Folgende ist die überarbeitete Fassung eines Essays, der in der Neuen Zürcher Zeitung vom 21./22.12.96 unter dem Titel „'Ich sah das Allerundenkbarste' - Der schreckliche Schatten schöner Literatur" veröffentlicht wurde.

1) Der Schatten. In: H. Ch. Andersen: Schräge Märchen - ausgesucht und übertragen von H. Detering. Frankfurt/M. 1996. S. 9-26. 
im heißen Land die Schatten und das Leben eigentlich erwachen, warf der Gelehrte mit Hilfe einer Zimmerlaterne seinen Schatten auf die Wand des geheimnisvollen Hauses auf der anderen Straßenseite, auf dessen Balkon sich selten genug eine wunderschöne Jungfrau gezeigt hatte. Auf ausdrücklichen Wunsch seines Herrn war der Schatten ins Haus gedrungen und nicht zurückgekehrt. Warum sollte er auch? Das Haus war nämlich nichts anderes als der Palast der Poesie. Die Poesie aber nimmt ganz offenbar keinen AnstoB daran, wenn in ihrem Kreis Wesen verkehren, die keinen Schatten oder keinen Schattenspender und also jene Double-Struktur nicht haben, an dem jede Vorstellung von Adäquanz oder Korrelation hängt. Im Palast der Poesie blieb der Schatten drei Wochen lang. Und in diesen drei Wochen wurde er zum Menschen - ja zu einem Übermenschen, da er mit schlechthin allem vertraut wurde, was es zu sehen und zu wissen gibt. "Ich sah alles, und ich weiß alles" ist seitdem die stehende Wendung desjenigen, der sich im Bann der Poesie von seinem Schattendasein emanzipierte.

$\mathrm{Ja}$, was sah er denn? Na, eben alles. Der Gelehrte wird betrogen. Der Schatten hat sich signifikant versprochen, als er versprach, nichts weniger als „alles" zu berichten. Denn „,alles" - nicht auszuschließen, daß diese Interpretation hier zu einem Schatten wird, der sich von der eigentlichen Geschichte emanzipiert - ,alles" läßt sich eben nicht aussagen. Sprechen läßt sich nur über „etwas“, nicht über ,alles". Deshalb gibt es Unaussprechliches. Und es gibt das Unaussprechliche gleich dreifach: als Erhaben-Unsagbares, als niederes Unaussprechliches und als funktional nicht Kommunizierbares. Der Gelehrte, der über das Gute, Wahre und Schöne schreibt, wird zwar nicht erfahren, was der Schatten alles sah, aber er muß erfahren, da $B$ der Schatten seine Allwissenheit und All-Einsicht recht pragmatisch genutzt hat. „Ich sah das Allerundenkbarste bei den Frauen, bei den Männern, bei den Eltern und bei den süßen, reinen Kindern." Man kann es auch weniger poetisch sagen: seinen Reichtum verdankt der Schatten seiner Kunst der sexuellen Erpressung, die niederes Unaussprechliches gleichwohl weitersagt.

Nichts ist hier leichter und stimmiger, als die Geschichte vom Mann, der seinen Schatten an eine Jungfrau verloren hat und der von diesem Verlust "gar nicht reden wollte“, psychoanalytisch zu deuten: als Geschichte einer homosexuellen Voyeur-Lust bzw. eines homosexuellen Verlustes. Bei Andersen liegt man damit fast immer richtig. Ein Mann hat seinen ständigen Begleiter, der ihn und den er deckte, an eine schöne Frau verloren. Was dieser im Reich der heterosexuellen Lust sah, kann er dem alten Freund unmöglich verständlich machen. Anspruchsvoller und aufschlußreicher als diese freudianische Deutung ist es allerdings, die sachlichfunktionale Variante des Unsagbarkeits-Topos zu betrachten. Denn Andersens Schatten-Geschichte stellt in klarer schwarz-weiß-Akzentuierung das 
alte, aber von klassischen Ästhetiken und Poetiken viel zu wenig bedachte Problem heraus, das jüngsı Niklas Luhmanns Untersuchung ..Die Kunst der Gesellschaft" in neuer systemtheoretischer Akzentuierung aufgerollt hat. Das so simple wie weitreichende Problem nämlich, dem das System Kunst und Literatur nichts Geringeres als seine Existenz und Funktionalität verdankt - das Problem. daß Wahrnehmung einfach nicht oder doch zumindest nicht einfach kommunizierbar ist. „Ich sah alles, und ich weiß alles." Aber ich sag's nicht. Weil es sich nicht sagen/schreiben/kommunizieren läßt.

Wer einmal versucht hat, einem Blinden den schlichten Unterschied zwischen blau und grün in dürren Worten mitzuteilen, wird schnell bereit sein zuzugestehen, daß Wahrnehmung nicht in Kommunikation et vice versa übersetzbar ist. Die Sprache der Literatur versucht sich dennoch an dieser Unmöglichkeit. In Luhmanns Worten: ..Kunst (ist) eine Art von Kommunikation, die ... Wahrnehmung in Anspruch nimmt." ${ }^{\text {I4 }}$ Und das. obwohl sie weiß. daß ,.das Bewußtsein ... nicht kommunizieren (und) die Kommunikation ... nicht wahrnehmen (kann). Übereinstimmungen bei so verschiedenen Operationsweisen müssen daher sehr abstrakt formuliert werden; denn es handelt sich um ganz verschiedene, je für sich operativ geschlossene, also überschneidungsfrei operierende Systeme. Mit Begriffen wie selbstreferentielles Ereignis. Unterscheidung. Form und Paradoxie haben wir die erforderliche Abstraktionslage erreicht. Dieser Hintergrund ermöglicht ... Rückschlüsse auf die Eigenart von Kunst. Kunst macht Wahrnehmung für Kommunikation verfügbar. und dies außerhalb der standardisierten Formen der (ihrerseits wahrnehmbaren) Sprache. Sie kann die Trennung von psychischen und sozialen Systemen nicht autheben. Beide Systemarten bleiben füreinander operativ unzugänglich. Und gerade das gibt der Kunst ihre Bedeutung. Sie kann Wahrnehmung und Kommunikation integrieren. ohne zu einer Verschmelzung oder Konfusion der Operationen zu führen."

Man kann es auch poetischer ausdrücken als Niklas Luhmann: Kunst (und ihr so eigentümliches, weil zugleich kommunikatives und antikommunikatives Double Literatur zumal) ist der lange Schatten, den das Problem der Nichtmitteilbarkeit und Nichtkommunizierbarkeit von Wahrnehmungen wirft. Denn schöne Literatur. die mit gutem Grund und offensiv die Stilmittel der literarischen Bildlichkeit in Anspruch nimmt, ist der stets erneut scheiternde und deshalb nicht endende Versuch. dieses Problem der Nichtmitteilbarkeit von Wahrnehmungen vergessen zu machen. Was (in Shakespeares ..Sommernachtstraum") Zettels Vision gewesen ist. kann keine noch so kunstvolle Ballade jemals ausdrücken. Allenfalls kann Sha-

it N. Luhmann: Die Kunst der Gesellschaft. Frankfurt/M. 1995. S. 26

"Ibid.. S. 82. 
kespeares Drama sich geistreich oder tiefsinnig darüber auslassen, wie komisch es ist, Wahrnehmungen dramatisch mitzuteilen und also auszudrükken, daß sich da etwas nicht kommunizieren läßt. „Zettel: Ich habe ein äuBerst rares Gesicht gehabt. Ich hatte 'nen Traum - 's geht über Menschenwitz, zu sagen, was es für ein Traum war. Der Mensch ist nur ein Esel, wenn er sich einfallen läßt, diesen Traum auszulegen. Mir war, als wär' ich - kein Menschenkind kann sagen, was. Mir war, als wär' ich, und mir war, als hätt' ich - aber der Mensch ist nur ein lustiger Hanswurst, wenn er sich unterfängt zu sagen, was mir war, als hätt' ich's; des Menschen Auge hat's nicht gehört, des Menschen Ohr hat's nicht gesehen, des Menschen Hand kann's nicht schmecken, seine Zunge kann's nicht begreifen und sein Herz nicht wieder sagen, was mein Traum war" (IV/I -Schlegel/Tieck-Übersetzung; Wieland radikalisiert hier noch stärker als die Romantiker den Witz Shakespeares, wenn er dessen Wendung ,at her (=Thisbes) death" mit "wenn ich mich erstochen habe" übersetzt).

Dies aber: daß sich Wahrnehmung nicht kommunizieren läßt, hat Zettel in synästhetisch deliranter Lust immerhin gesagt. Und er hat es so dicht, schön und kratylistisch unfehlbar gesagt, daß wir zu recht bereit sind. diese Passage als eine der großen der Weltliteratur zu akzeptieren. Zumal sie ja auch analytisch klar fortfährt - nämlich mit dem Hinweis auf Anschlußmöglichkeiten beim Versuch, das Problem der Nichtkommunizierbarkeit von Wahrnehmung zu lösen, indem man es bewahrt. „Ich will den Peter Squenz dazu kriegen, mir von diesem Traum eine Ballade zu schreiben: sie soll Zettels Traum heißen, weil sie so seltsam angezettelt ist. und ich will sie gegen das Ende des Stücks vor dem Herzog singen. Vielleicht, um sie noch anmutiger zu machen, werde ich sie nach dem Tode singen." In 'Zettels Traum Werk, das wie kein zweites im 20. Jahrhundert seinen Reiz daraus bezieht, der Literatursprache Krisen zu verschreiben, hat Arno Schmidt getan, was Peter Squenz unterlassen hat.

Arno Schmidts opus magnum macht auch mit wünschenswerter Entschiedenheit deutlich, daß die Sprache der Literatur eben nicht kommunikativ, sondern antikommunikativ ist: sie erschwert Verständigung, sie wirft Schatten, sie lenkt ab, sie verschiebt systematisch Bedeutungen, sie spielt mit Doubles, sie kommuniziert über metaphorische und metonymische Ecken. Und das heißt: sie fürchtet nichts so sehr wie den Schein schattenlos-direkter Kommunikation. Warum? Weil, mit Lacan zu formulieren, etwas nicht aufhört. sich nicht zu schreiben. Und wenn es doch aufhört, sich nicht zu sagen, zu schreiben und zu lichten, dann um einen ungeheuren Preis: den der apokalyptisch strahlenden Klarheit, die alle Schatten vergehen oder eben alles zu Schatten werden läßt.

Was sein Ex-Schatten gesehen hat und was er weiß, wird der gelehrte Herr aus Andersens Märchen nie erfahren. Er grämt sich darüber und über 
den Umstand, daß die Welt am Wahren, Guten und Schönen gar kein Interesse hat, so sehr, daß er zum Schatten seiner selbst wird. Jahre später kommt sein alter, nun fast schon dick gewordener Schatten wieder. Er, der schattenlose Ex-Schatten, sucht nämlich einen Reisebegleiter für die Zeit seiner Kur, die seine Bartlosigkeit (und also ein männliches Defizit) kurieren soll - er sucht einen Kurschatten. Der Gelehrte sagt zu. „Der Schatten war nun Herr, und der Herr war Schatten", wie es in Andersens seltsam schattenlos-direkter Prosa heißt. Doch das Verlustdrama wiederholt sich. Statt sich über die Wiedervereinigung und die neue Rollenverteilung zu freuen, geht der Ex-Schatten fremd. Er gewinnt das Herz einer „wunderschönen Königstochter, die an der Krankheit litt, daß sie allzu scharf sehen konnte" und die also sieht, daß nicht die Bart-, sondern die Schattenlosigkeit das Problem ihres Galan ist. Deshalb verweist der auf seinen exklusiven, nämlich menschlichen Schatten. Der Gelehrte muß sich buchstäblich unterwerfen und als Schatten seinem Herrn zu Füßen liegen. Der Königstochter gefällt das offenbar, und der Hochzeit steht nichts mehr im Wege. Doch - der gedemütigte Mensch/Schatten droht, ,alles" zu sagen. Aber das geht einfach nicht: er wird hingerichtet. Der zum Schatten seiner selbst gewordene Mensch wird als Schatten in die Hadesregionen eingehen.

Der Mensch/Schatten aus Andersens bester (präziser: wohl einzigen wirklich guten Geschichte) wird sich demnach einreihen in die lange Ahnenreihe derer, die zu Schatten wurden. Andersens Geschichte ist ein Schatten. Sie steht deutlich im Schatten nicht nur der berühmtesten Schatten-Geschichte: der Geschichte Peter Schlemihls. Sie wird auch von den vielen weiteren Schatten-Geschichten, die ihr vorangegangen sind. in den Schatten gestellt. Sie steht im Schatten des platonischen Höhlengleichnisses; im Schatten von Antigones großer Abschiedsklage an die untergehende Sonne über Theben, die sie ein letztes Mal sieht, bevor sie ins Reich der Schatten hinab muß; im Schatten von Orpheus und Euridice; im Schatten Amphitryons und seines göttlichen Doubles und und und. Bis die Aufzählung zu Hofmannsthals "Frau ohne Schatten“ oder zu Peter Weissens „Der Schatten des Körpers des Kutschers" vorgedrungen sein wird, wird sie an schattiger Länge alle zumutbaren Größenordnungen überschritten haben. Alle Schatten auch nur der wirklich bedeutenden Literatur aufzählen $z u$ wollen hieße diesen kleinen Vortrag zum (bestenfalls blutlos gelehrten) Schatten seiner selbst zu machen.

Große Literatur lebt von der Differenz zu anderer großer Literatur. Sie will mehr sein als nur deren Schatten. Umso bemerkenswerter ist es deshalb, daß so gut wie alle Schatten-Geschichten von Wahrnehmungs- und Mitteilungsdefiziten oder aber eben von allzu scharfer Wahrnehmung berichten. Ihnen fällt auf, daß zwischen Wahrnehmung und Kommunikation ein Abgrund klafft. Und kluger Literatur fällt eben nicht nur auf, daß auch 
ein Licht sein muß, wo Schatten ist, sondern daß geblendet werden kann, wer dieses Licht unmittelbar ohne den filternden Schutz von Schatten sehen will. „Alles“ läßt sich nicht sagen und schreiben. Weil wir das Licht nicht unmittelbar schauen können, weil jede vollendete Offenbarung auch eine explosive Apokalypse wäre, deshalb sind binär prozedierende Theoriekonzepte, die Licht und Schatten aufeinander beziehen, so unwiderstehlich. Alle großen Weltanschauungen, Erzählungen und Theorien arbeiten mit Differenzbegriffen, die einander zugeordnet sind wie Licht und Schatten. Schlechthin alle fundierenden Terme der Theoriegeschichte führen einen Schatten- und Komplementärbegriff mit sich.

Mit der eigentümlichen Unterscheidung zwischen Hell und Dunkel bzw. dem eigentlichen Sein der Ideen und dem uneigentlichen Sein des Materiellen hatte die Geschichte der Großtheorien ihren platonischen Startpunkt gefunden. Mit der theologischen Urunterscheidung von Licht und Schatten, von Gut und Böse, von Gott und Teufel war diese platonische Grundgeste zwanglos assoziierbar. Draw a distinction, unterscheide Primäres von Sekundärem, ordne Licht und Schatten einander zu! Viel weiter als die religiös-platonische Tradition ist auch das avancierte Theoriedesign bislang nicht gekommen. Wir denken bis heute (fast) unabweisbar in binären Schemata und Begriffen: das Unbewußte ist der Schatten des Bewußten, die Basis der des Überbaus, der Signifikant der des Signifikats, Anima der von Animus, die Umwelt der des Systems, das Nichts der des Seins etc. Modern sind Theorien dann, wenn sie eine Umwertung der eingespielten Zuordnungen von Licht- und Schattenbegriffen versuchen. Die Geschichte neuerer Theoriebildungen ab 1850 ist zumeist die Geschichte solcher Umwertungen aller bisherigen Bewertungen. Mit Marx kann man dann argumentieren, die Basis sei bedeutender als der Überbau; mit Nietzsche, das Nichts sei mächtiger bzw. eigentlicher als das Sein; mit Freud, das Unbewußte sei der eigentliche Herr im Haus des Ich; mit de Saussure, der Signifikant dominiere das Signifikat etc.

Das Spiel der Umwertungen ist oder war doch zumindest reizvoll. Vom alten Denkzwang, es müsse verläßliche Zuordnungen zwischen Wahrnehmung und Kommunikation, zwischen Sein und Zeichen, zwischen Sachen und Wörtern geben, haben wir uns zwar verabschiedet. Allzu klar machen Schatten deutlich, daß sie je nach der Beobachterperspektive und der Lichtquelle, der sie sich verdanken, kürzer oder länger (aus)fallen können als das, wovon sie Schatten sind. Vielleicht sind postmoderne Theorien einen kleinen Schritt weiter gelangt. Sie machen es möglich, nicht nur Licht und Schatten zu unterscheiden, sondern auch die Funktion dieser Unterscheidung selbst zu beobachten. Sie schalten, um im systemtheoretischen Jargon zu reden, auf second-order-observation um: sie beobachten nicht nur (wie der Schatten des Gelehrten), sondern sie beobachten 
Beobachtungen. Z.B. die Beobachtung von Licht-/Schatten-, Wahrnehmung/Kommunikation- oder Sprache/Literatursprache-Differenzen. Sie können dabei zu so abgründigen Schlußsätzen wie dem aus Kafkas, Gespräch mit dem Beter ‘ kommen: „Und Geständnisse würden am klarsten. wenn man sie widerriefe." 